\title{
Water quality assessment based on CWQI and NDWI indices in Mariout Lake, Egypt
}

\begin{abstract}
Alexandria Governorate inhabited by 4 million people is located on the north western edge of the Nile Delta. It extends about 70 kilometers north-west of the Nile Delta. Lake Mariut, one of Egypt's Northern Lakes facing the Mediterranean Sea, is located within the Alexandria Governorate Borders. Since the last four decades. Lake Mariut falls under enormous pressure of un-planned development, pollution and land reclamation. Now-a-day, Lake Mariut suffers from pollution, reduction of its surface area, un-planned development in a way that considerable part of the lake entered into possible irreversible processes. The purpose of this study is to assess the degree of pollution in the context of CWQI (Canadian Water Quality Index) in Mariut Lake by determining various physicochemical parameters, as the concentration, PH, DO, BOD, CHL-a, TSM, NH4, TP, and NO3 during from 2010 to 2014._Using CWQI found that the water quality of the studied Lake is found according to CWQIs model which indicates that water quality of this Lake is moderate and frequently impaired for determining water quality and its area constantly decreased according to NDWI. Results of this research showed that there are large variations in parameters in different sections, and these variations are affected by different factors. Such pressures deteriorated the Lake Mariut environmental resources quality, decreased it surface area and created problems to the Large Marine Ecosystem (Eastern Mediterranean Part). The study concluded that the area of the Lake Mariut was exposed to multiple stages of drying in order to create economic development activities for the community of Alexandria Governorate. The impact of sewage disposal to parts of the Lake basins was quite obvious too.
\end{abstract}

Keywords: water resource management, water quality indexes, water pollution, Mariout Lake, NDWI, remote sensing, GIS applications
Volume 2 Issue 5 - 2017

\author{
Sameh B El Kafrawy, ' Noha S Donia, ${ }^{2}$ Amany \\ Morsi Mohamed' \\ 'National Authority for Remote Sensing and Space Sciences \\ (NARSS), Egypt
}

${ }^{2}$ Institute of Environmental Studies and researches (ASU), Egypt

Correspondence: Sameh B El Kafrawy, National Authority for Remote Sensing and Space Sciences (NARSS), Egypt, Email sameh@narss.sci.eg.s_elkafrawy@yahoo.com

Received: June 15, 2017| Published: August 08, 2017
Abbreviations: NDWI, the normalized difference water index; CWQI, Candian water quality index; WQIA, water quality index; WQIM, multiplicative water quality index; WQIUA, un-weighted arithmetic water quality index; WQIH, Harkins' water quality index

\section{Introduction}

Water Quality Index, a critical tool for water resources management, will be developed to evaluate and communicate the suitability of water bodies in Egypt for various uses such as drinking, irrigation, livestock, aquatic life, and recreation. ${ }^{1}$ The CCME WQI provides a measure of the deviation of water quality from water quality guidelines. The CCME WQI model consists of three measures of variance from selected water quality objectives (Scope; Frequency; Amplitude). These three measures of variance combine to produce a value between 0 and 100 that represents the overall water quality. The CCME WQI values are then converted into rankings by using an index categorization schema that can be customized to reflect the expert opinion by users. The detailed formulation of the WQI is described in the Canadian Water Quality Index 1.0 - Technical Report (CCME 2001). ${ }^{1}$

\section{Study area and methodology}

\section{Study area}

Lake Mariout is a salt lake of about 250 square $\mathrm{km}$ in northern Egypt, lies between Latitude $31^{\circ} 01$ ' $48^{\prime \prime}$ and 310 10'30" $\mathrm{N}$ and Longitude 29 $57^{\prime}$ '00" E along the Mediterranean coast of Egypt. It forms the southern boundary of the city of Alexandria. It is a closed lake with a surface area of $60 \mathrm{~km} 2$ and ranges in depth from 1-3m. The lake is divided into 4 basins. These are the main (6000 acres), South West (5000 acres), North West (3000 acres), and aquaculture or fisheries (1000 acres). The lake is fed by canals from the Rosetta branch of the Nile. Several branches of these canals allow water flow from and to these basins. The main canals are al Kalaa drain, Omum drain and Nubaria canal. The water inflow to the lake came mainly from these sources plus those from a Wastewater treatment plant, the discharge from the west Nubaria drain and from the petrochemical area. Outflow from the lake is only released by El-Max pumping station. Domestic sewage is discharged from the wastewater treatment plant at the north and main basin and with the El-Kalaa drain to the lake body. Thus, the lake acts as a receptor for agricultural drainage and domestic discharges as well as a source of irrigation water to the nearby cultivated lands. This resulted in severe pollution in the main basin. The discharge from Omum and Nubaria canals, though less polluted, it contains pesticides and fertilizers besides raw and treated wastewater. It seems that the lake has been abused and misused severely resulted in a highly deterioration and pollution. The huge amounts of wastewater from different sources around the lake (west and east sewerage plants), industrial settlements, factories, workshops, plus agricultural drainage contribute to the deterioration of the water quality of the lake. Consequently, the lake becomes eutrophic where the excessive growth of algae and larger aquatic plants takes place leading to the consumption of dissolved oxygen. The light penetration in this shallow water lake and excessive nutrients from sewage, fertilizers and industrial wastes contribute in the eutrophication of the lake (Figure 1) (Table 1). 


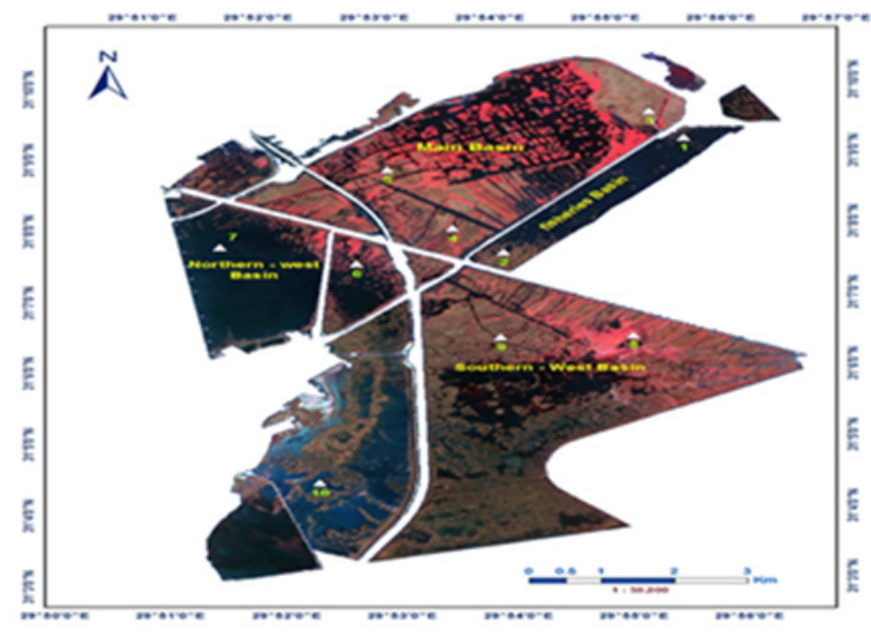

Figure I Lake Mariout.
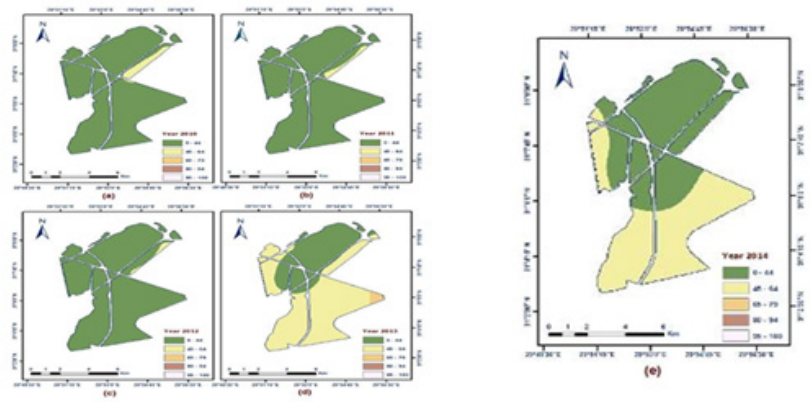

Figure 2 Spatial Interpolation Maps of CWQI in Mariout Lake Water. (a) 2010 , (b) 20II, (c) 20I2, (d) 20I3, and (e) 2014.

\section{Methodology}

One of pollution assessment and quality ranging methods of lakes is using a quality index which shows the compound effect of physicchemical and biological parameters. There follows an explanation of water quality indices applied in this research. Assessment of surface water quality can be a complex process undertaking multiple parameters capable of causing various stresses on overall water quality. Evaluation of water quality from a large number of samples, each containing concentrations for many parameters is difficult. Depending on the availability of data, analysis has been carried out using CWQI for spatial variation. The data analysis carried out to calculate Water Quality Indices such as CWQI is explained below.

EEAA Field data are essential to design Candian Water Quality Index (CWQI) for Mariout Lake. To obtain such data, ten different sampling locations were selected. These locations were chosen on the basis of maximum waste flow to the lake. Water samples were collected at Three months in the year 2010, 2011, 2012, 2013 and 2014. There are Various Water Quality Indices such as Arithmetic Water Quality Index (WQIA), Multiplicative Water Quality Index (WQIM), Un-weighted Arithmetic Water Quality Index (WQIUA), Harkins' Water Quality Index (WQIH), Delphi Approach for Water Quality Index Calculation, The British Columbia Index, and Canadian Water Quality Index. CWQI is suggested to be a helpful tool that enables the public and decision makers to evaluate water quality of lakes, CWQI was calculated to assess the spatial and temporal variability and identify the classification of water quality in the study area. In order to simplify the procedure and reduce the analytical costs of the water quality evaluation, similar spatial changing trend and classification of the water quality, the results also suggest that continuous monitoring should be conducted to prevent pollution from industry and anthropogenic activities. The CWQI of study area ranged from 13.13 to 63.42 . Hence the lake water quality is among poor and marginal.

Table I Selected stations, common names and their latitudes and longitude in Mariout Lake

\begin{tabular}{|c|c|c|c|c|}
\hline Station No. & location & Basin & Latitude (North) & Longitude (East) \\
\hline I & The first farm I000 Feddan (shadir fish) & Fisheries & 31009112.611 & $29055 \backslash 37.911$ \\
\hline 2 & last farm I000 Feddan (Alhbact) & & $31007 \backslash 37.611$ & $29054 \backslash 01.811$ \\
\hline 3 & In front of the Qalaa drainge & Main & $31009 \backslash 35.311$ & $29055 \backslash 20.64 \backslash 1$ \\
\hline 4 & Northeast the Bridge Abul Khair & & $31007 \backslash 58.111$ & $29053 \backslash 35.521 \backslash$ \\
\hline 5 & Half of Basin 3000 Feddan & & $31008 \backslash 48.5 \backslash \backslash$ & $29053 \backslash 03.1211$ \\
\hline 6 & In Front of Max Pumps & & $31007 \backslash 29.311$ & 29052145.1211 \\
\hline 7 & First Basin 5000 Feddan & Southwest & $31007 \backslash 44.811$ & $29051 \backslash 34.5611$ \\
\hline 8 & Last Basin 5000 Feddan & & $31006 \backslash 25.911$ & $29055 \backslash 06.611$ \\
\hline 9 & In front of the Western purification plant exchange & Northwest & $31006 \backslash 25.911$ & $29053 \backslash 57.84 \backslash 1$ \\
\hline 10 & Central Basin 2000 Feddan & & $31004 \backslash 24.611$ & 29052120.2811 \\
\hline
\end{tabular}

Table 2 Range of CCME-WQI (Canadian Environmental Quality Guidelines, 1999,Winnipeg)

\begin{tabular}{|c|c|c|}
\hline Rank & WQI value & Description \\
\hline Excellent & $95-100$ & Water quality is intact; conditions are very close to natural or desired levels \\
\hline Good & $80-94$ & $\begin{array}{l}\text { Water quality is intact; and only one minor threat or deterioration is observed, conditions rarely differed from the } \\
\text { natural or desirable level. }\end{array}$ \\
\hline Fair & $65-79$ & $\begin{array}{l}\text { Water quality is usually intact, but occasionally endangered or deteriorated; conditions sometimes deviate from natural } \\
\text { or desirable levels. }\end{array}$ \\
\hline Marginal & $45-64$ & Water quality is frequently endangered or deteriorated. Conditions often deviate from natural or desirable levels. \\
\hline Poor & $0-44$ & Water quality is always endangered or deteriorated; conditions usually deviate from natural or desirable levels. \\
\hline
\end{tabular}

Source: CCME (200I). 


\section{Calculation of Canadians Water Quality Index (CWQI)}

Canadian water quality index was founded in 1995 based on water quality index, released by British Columbia University. A merit of this index is using numerous quality variables for calculating this index, though it has some limitations as well. The Canadians Water Quality Index (CWQI) is calculated using the Canadian Council of Ministers of the Environment Index method. Following expressions are used to determine the CWQI for our study. Three factors are combined to calculate the CWQI, F1 (Scope), F2 (Frequency) and F3 (Amplitude) F1 (scope) represents the percentage of variables that do not meet their objective at least once during the time period under consideration ("failed variables"), relative to the total number of variables measured:

$$
F_{1}=\left(\frac{\text { Number of failed variables }}{\text { Total number ofvariables }}\right) \times 100
$$

F2 (Frequency) represents the percentage of individual tests that do not meet objectives ("failed test"):

$$
F_{2}=\left(\frac{\text { Number of failed tests }}{\text { Total number of tests }}\right) \times 100
$$

$\mathrm{F}_{3}$ (Amplitude) represents the amount by which failed test values do not meet their objectives. F3 is calculated in three steps.

The number of times by which an individual concentration is greater than (or less than, when the objective is a minimum) the objective is termed an "excursion" and is expressed as follows: When the test value must not exceed the objective:

$$
\text { excursion }_{i}=\left(\frac{\text { Failed test value }}{\text { Objective }_{j}}\right)-1
$$

For the cases in which the test value must not fall below the objective:

$$
\text { excursion }_{j}=\left(\frac{\text { Objective }_{j}}{\text { Failed test value }_{\text {ele }}}\right)-1
$$

The collective amount by which individual tests are out of compliance is calculated by summing the excursions of individual tests from their objectives and dividing by the total number of tests (both those meeting objectives and those not meeting objectives). This variable, referred to as normalized sum of excursions, or nse, is calculated as: F3 is then calculated by an asymptotic function that scales the normalized sum of the excursions from objectives (nse) to yield a range between 0 and 100 .

$$
\begin{aligned}
& \text { nse }=\frac{\sum_{i=1}^{n} \text { excursion }_{i}}{\text { number of tests }} \\
& F_{3}=\left(\frac{n s e}{0.01 \text { nse }+0.01}\right)
\end{aligned}
$$

Once the factors have been obtained, the index itself can be calculated by summing the three factors as if they were vectors. The sum of the squares of each factor is therefore equal to the square of the index. This approach treats the index as a three-dimensional space defined by each factor along one axis. With this model, the index changes in direct proportion to changes in all three factors.

$$
C W Q I=100-\frac{\sqrt{ } F_{1}^{2}+F_{2}^{2}+F_{3}^{2}}{1.732}
$$

The CWQI value ranges between 1 and 100, the result is further simplified by assigning it to a descriptive category as Table $1 \&$ Table 2.

\section{Results and discussion}

\section{Water quality parameters}

For the selected water quality parameters, general information, environmental effects and the different pollutions sources (Tables 3-6 \& Figures 2-4). 3,4
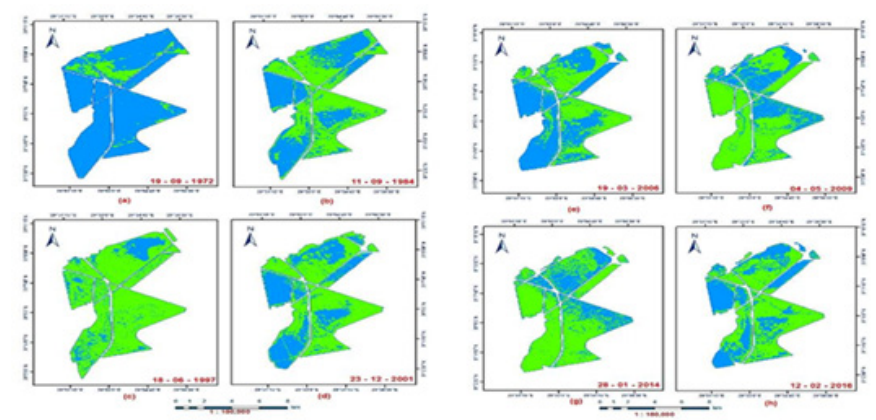

Figure 3 NDWI Images of the study area (a) 1972, (b) 1984, (c) 1997, (d) 200 I (e) 2006, (f) 2009, (g) 2014, (h) 2016.



Figure 4 NDWI Changes of Mariout Lake during 1972 to 2016.

$\mathrm{PH}$ ranged from 7.1 to 9.17 , Station 3 denoted low $\mathrm{PH}$ (7.10). This might relate to the activity of bathing, washing, and latrines along the water bodies. The residue from those activities carried organic material and was decomposed by microorganisms. The process also consumed oxygen and released carbon dioxide. Hence, the water $\mathrm{pH}$ tended to acidic. EEAA quality standards requires $\mathrm{pH}$ of 6.5- 8.5; therefore the station $10, \mathrm{PH}$ was out of the quality standard.

Dissolved oxygen (DO) is the concentration of oxygen dissolved in the water. Its presence is essential to maintain the variety of biological life forms in water and the effect of the waste discharge in a water body is largely determined by oxygen balance of the system. ${ }^{5}$ Natural water bodies have high levels of oxygen which vary depending on temperature, salinity, water turbulence, and atmospheric pressure. ${ }^{6}$ The colder the water, the more oxygen it can hold. ${ }^{7} \mathrm{DO}$ ranged from 0 to $15.8 \mathrm{mg} / \mathrm{l}$. Dissolved oxygen is necessary for the survival of aquatic organisms. The low DO indicates high demand for oxygen by the microorganisms, ${ }^{8}$ The more organic materials that pollute the water body, the more amount of oxygen consumed to decompose 
these materials so that the content of dissolved oxygen in the water decreases so low. ${ }^{9}$ EEAA quality standards, DO must be $>=5 \mathrm{mg} / 1$, hence Station 8 denoted high DO (15.8), but at the station 3, DO was out of the quality standard.

Biochemical Oxygen Demand (BOD) is a measure of the amount of oxygen used by microorganisms to decompose organic materials in water within five days period. ${ }^{10}$ Low BOD content indicated that the riverside stretch was free from organic pollution. ${ }^{5}$ The BOD in Mariout lake ranging $1.16-729 \mathrm{mg} / \mathrm{l}$. Station 4 denoted low BOD (1.16), and station 3 is exceeded the quality standard. This might due to the decomposition process of organic matter by microbes consuming oxygen. High BOD in water is undesirable because it will reduce the DO. ${ }^{11}$ EEAA quality standards, BOD must be $<6 \mathrm{mg} / 1$.

Chlorophyll-a gives plants their green colour and is produced by microscopic plants called algae in lakes. The amount of chlorophyll-a in water ranged $0.86-347.86 \mathrm{ug} / \mathrm{l}$, it was highest in Station 10 (347.86ug/l) and lowest in station $2(0.86 \mathrm{ug} / \mathrm{l})$ because of course it is not easy for plants to grow. There are many human activities that affect chlorophyll in water, such as sewage inputs and destruction of lake. EEAA quality standards requires Chlorophyll-a $<=140 \mathrm{ug} / 1$; therefore most stations were out of the quality standard.
Total suspended matter (TSM) ranged 11.93-114.03. TSM transports nutrients and contaminants, reduces light transmission through a water column and influences entire aquatic ecosystems. ${ }^{12}$ Accurate spatiotemporal information on TSM distribution patterns is therefore important for understanding Lake Ecosystem dynamics and for the development of effective and quantitative monitoring of aquatic environments, in order to both protect the lake ecosystem and improve water quality management. ${ }^{13-15}$ Station 8 recorded low TSM (11.93), and Station 10 recorded highest TSM (114.03). EEAA quality standards require TSM of 25; therefore the most stations, TSM were out of the quality standard.

Ammonia levels ranging 0.05 - $20.34 \mathrm{mg} / \mathrm{l}$, Stations $(1,2$ and 10) recorded low Ammonia (0.05). This was likely due to the small content of urea and ammonification process derived from the decomposition of organic matter by microbes. Since agricultural activities is still managed in a traditional means. The small number of residential area also caused the low level of ammonia. The presence of ammonia is an evidence of sewage inflow to a water body.$^{16}$ Domestic and industrial wastewater constitutes a constant polluting source. The ammonia recorded high at station 3 was $20.34 \mathrm{mg} / 1$, the lake water is considered polluted. EEAA quality standards, ammonia content must be $\leq 0.41 \mathrm{mg} / 1$, hence ammonia of the most observations was out of the quality standard.

Table 3 Water quality parameters of 10 stations for the year $2010,2011,2012,2013,2014$

\begin{tabular}{|c|c|c|c|c|c|c|c|c|c|}
\hline $\begin{array}{l}\text { Station } \\
\text { No. }\end{array}$ & $\begin{array}{l}\text { Sample } \\
\text { date }\end{array}$ & $\begin{array}{l}\text { PH (6.5- } \\
8.5)\end{array}$ & $\begin{array}{l}\mathrm{DO}(>5) \\
\mathrm{mg} / \mathrm{l}\end{array}$ & $\begin{array}{l}\text { BOD (3- } \\
\text { 6) } \mathrm{mg} / \mathrm{l}\end{array}$ & $\begin{array}{l}\text { CHL-A (5- } \\
\text { 140) ug/I }\end{array}$ & $\begin{array}{l}\text { TSM } \\
(25) \mathrm{mg} / \mathrm{l}\end{array}$ & $\begin{array}{l}\mathrm{NH} 4(0.4 \mathrm{I}) \\
\mathrm{mg} / \mathrm{I}\end{array}$ & $\begin{array}{l}\mathrm{NO} 3(<0.1) \\
\mathrm{mg} / \mathrm{l}\end{array}$ & $\begin{array}{l}\text { TP (25- } \\
\text { 100) ug/I }\end{array}$ \\
\hline \multirow[t]{3}{*}{ I } & Feb.20I0 & 8.4 & 7.73 & 14.05 & 8.47 & 39.8 & 5.22 & 0.45 & 54.52 \\
\hline & May.20I0 & 8.36 & 7.8 & 7 & 42.26 & 65.75 & 0.57 & 0.11 & 100.25 \\
\hline & Aug.2010 & 8 & 7.26 & 1.5 & 9.04 & 36.05 & 0.78 & 0.59 & $|20.7|$ \\
\hline \multirow[t]{3}{*}{2} & Feb.20I0 & 8.43 & 7.67 & 11.62 & 6.96 & 40.5 & 4.4 & 0.49 & 57.75 \\
\hline & May.2010 & 8.35 & 7.04 & 5.8 & 12.35 & 50.65 & 0.59 & 0.04 & 79.79 \\
\hline & Aug.2010 & 8.3 & 7.76 & 16.2 & 8.82 & 41.4 & 0.6 & 0.19 & 67.52 \\
\hline \multirow[t]{3}{*}{3} & Feb.20I0 & 7.53 & 0.04 & 21.49 & 1.26 & 37 & 9.42 & 0.01 & 568.72 \\
\hline & May.2010 & 7.6 & 0 & 28 & 2.09 & 37 & 18.66 & 0.04 & 3470.02 \\
\hline & Aug.2010 & 7.1 & 0.09 & 47.2 & 1.69 & 51.55 & 4.88 & 0.19 & 2185.13 \\
\hline \multirow[t]{3}{*}{4} & Feb.20I0 & 7.58 & 4.35 & 11.34 & 1.26 & 20.9 & 4.73 & 3.66 & 214.83 \\
\hline & May.2010 & 8.56 & 3.2 & 5.98 & 72.53 & 31.88 & 1.13 & 1.49 & 431.71 \\
\hline & Aug.2010 & 7.7 & 1.83 & 7.4 & 1.69 & 31.25 & 0.97 & 1.4 & 353.96 \\
\hline \multirow[t]{3}{*}{5} & Feb.20I0 & 8.81 & 11.66 & 36.05 & 64.73 & 22.4 & 4.65 & 0.36 & 113.65 \\
\hline & May.2010 & 7.95 & 1.6 & 9.8 & 287.17 & 47.35 & 7.4 & 0.17 & 2269.01 \\
\hline & Aug.2010 & 8.5 & 8.8 & 3 & 9.34 & 62.15 & 0.54 & 0.15 & 196.42 \\
\hline \multirow[t]{3}{*}{6} & Feb.2010 & 7.91 & 0.35 & 10.67 & 7.01 & 46.7 & 7.57 & 0.13 & 465.7 \\
\hline & May.20I0 & 8.4 & 11.2 & 6.4 & 268.89 & 64.78 & 0.71 & 0.64 & 370.33 \\
\hline & Aug.2010 & 8 & 6.92 & 13.3 & 78.32 & 51.2 & 0.62 & 1.06 & 149.36 \\
\hline \multirow[t]{3}{*}{7} & Feb.20I0 & 7.5 & 9.46 & 3.95 & 74.47 & 40 & 5.2 & 1.06 & 101.64 \\
\hline & May.2010 & 8.39 & 8.2 & 7.2 & 123.77 & 54.18 & 0.52 & 0.11 & 118.67 \\
\hline & Aug.2010 & 7.5 & 2.31 & 10.3 & 79.44 & 27.3 & 0.82 & 1.09 & 290.53 \\
\hline \multirow[t]{3}{*}{8} & Feb.20I0 & 7.6 & 5.07 & 2.16 & 1.64 & 16.6 & 5.15 & 0.33 & 189.42 \\
\hline & May.20I0 & 8.53 & 8 & 6 & 171.73 & 57.28 & 0.51 & 0.81 & 233.24 \\
\hline & Aug.2010 & 7.4 & 2.2 & 5.9 & 1.75 & 20.6 & 0.58 & 1.69 & 288.49 \\
\hline \multirow[t]{3}{*}{9} & Feb.20I0 & 8.4 & 10.6 & 14.05 & 1.64 & 15 & 5.32 & 0.74 & 55.44 \\
\hline & May.2010 & 8.32 & 6.82 & 6.14 & 6.28 & 19.13 & 0.51 & 0.11 & 45.01 \\
\hline & Aug.2010 & 7.2 & 0.09 & 47.2 & 2.21 & 79.6 & 5.86 & 0.24 & 226.83 \\
\hline \multirow[t]{3}{*}{10} & Feb.20I0 & 8.2 & 10.19 & 11.62 & 5.71 & 33.6 & 4.89 & 1.19 & 44.81 \\
\hline & May.2010 & 8.31 & 12.4 & 4.6 & 347.86 & 148.03 & 0.78 & 0.64 & 226.83 \\
\hline & Aug.2010 & 8 & 6.38 & 16.2 & 6.09 & 51.7 & 0.5 & 0.39 & 226.83 \\
\hline
\end{tabular}




\begin{tabular}{|c|c|c|c|c|c|c|c|c|c|}
\hline $\begin{array}{l}\text { Station } \\
\text { No. }\end{array}$ & $\begin{array}{l}\text { Sample } \\
\text { Date }\end{array}$ & $\begin{array}{l}\text { PH (6.5- } \\
8.5)\end{array}$ & $\begin{array}{l}\mathrm{DO}(>5) \\
\mathrm{mg} / \mathrm{l}\end{array}$ & $\begin{array}{l}\text { BOD (3- } \\
6) \mathrm{mg} / \mathrm{l}\end{array}$ & $\begin{array}{l}\text { CHL-A (5- } \\
\text { I40) ug/l }\end{array}$ & $\begin{array}{l}\text { TSM(25) } \\
\mathrm{mg} / \mathrm{l}\end{array}$ & $\begin{array}{l}\text { NH4 } \\
(0.4 I) \mathrm{mg} / \mathrm{l}\end{array}$ & $\begin{array}{l}\mathrm{NO3}(<0.1) \\
\mathrm{mg} / \mathrm{l}\end{array}$ & $\begin{array}{l}\text { TP }(25-100) \\
\text { ug/l }\end{array}$ \\
\hline \multirow[t]{3}{*}{ I } & Feb.20II & 9.1 & 10.25 & 4.54 & 41.82 & 32.5 & 3.2 & 0.21 & 61.38 \\
\hline & May.20II & 8.5 & 8.51 & 17.16 & 8.64 & 46.4 & 0.13 & 0.06 & 90.02 \\
\hline & Aug.201I & 8.31 & 6.54 & 25.34 & 23.78 & 125.2 & 0.65 & 0.32 & 24.55 \\
\hline \multirow[t]{3}{*}{2} & Feb.20II & 9 & 12.13 & 10.4 & $|4.5|$ & 34.6 & 0.2 & 0.43 & 106.39 \\
\hline & May.20II & 8.5 & 8.34 & 13.02 & $5.4 I$ & 42.35 & 0.11 & 0.16 & 73.66 \\
\hline & Aug.20II & 8.41 & 6.87 & 15.53 & 65.93 & 108.65 & 0.71 & 0.1 & 49.1 \\
\hline \multirow[t]{3}{*}{3} & Feb.20II & 7.5 & 0.77 & 21.82 & 0 & 49.4 & 3.33 & 1.21 & 652.67 \\
\hline & May.20II & 7.8 & 0 & 84 & 5.86 & 104.75 & 16.53 & 0.05 & 554.47 \\
\hline & Aug.20II & 8.11 & 0 & 106.28 & 10.73 & 38.2 & 6.86 & 0.09 & 581.06 \\
\hline \multirow[t]{3}{*}{4} & Feb.20II & 7.6 & 3.16 & 13.4 & 4 & 32.4 & 4.05 & 2.83 & 470.58 \\
\hline & May.20II & 7.8 & 2.67 & 1.16 & 17.18 & 33.5 & I.I7 & 0.81 & 570.83 \\
\hline & Aug.20II & 8.22 & 4.74 & 5.72 & 11.61 & 48.85 & 0.84 & 0.4 & 310.99 \\
\hline \multirow[t]{3}{*}{5} & Feb.20II & 7.7 & 4 & II. 27 & 11.77 & 17.35 & 3.65 & 1.92 & 431.71 \\
\hline & May.20II & 8.2 & 4.37 & 6.11 & 72.28 & 30.4 & 3.01 & 0.14 & 127.47 \\
\hline & Aug.20II & 8.52 & 3.27 & 10.63 & 70.87 & 66.05 & 2.73 & 1.18 & 838.86 \\
\hline \multirow[t]{3}{*}{6} & Feb.20II & 7.8 & 1.31 & 14.54 & 11.72 & 31.05 & 4.76 & I.7I & 611.75 \\
\hline & May.20II & 7.6 & 0 & 38.56 & 14.56 & 37.6 & 3.23 & 0.69 & 1106.89 \\
\hline & Aug.201 I & 8.08 & 1.8 & 55.59 & 33.68 & 133.45 & 5.53 & 0.17 & 1196.91 \\
\hline \multirow[t]{3}{*}{7} & Feb.20II & 8.7 & 11.63 & 8.1 & 5.75 & 57.85 & 3.43 & 0.24 & 106.39 \\
\hline & May.20II & 8.5 & 15.07 & 29.27 & 14.56 & 37.6 & 3.23 & 0.69 & 1106.89 \\
\hline & Aug.201I & 8.53 & 4.58 & 31.88 & 12.42 & 88.25 & 0.38 & 0.11 & 104.35 \\
\hline \multirow[t]{3}{*}{8} & Feb.20II & 7.8 & 6.85 & 6.24 & 9 & 32.85 & 3.96 & 2.66 & 454.21 \\
\hline & May.20II & 8.4 & 15.8 & 31.7 & 47.7 & 56.95 & 0.17 & 0.1 & 218.92 \\
\hline & Aug.201I & 8.34 & 4.91 & 35.15 & 7.49 & 36.55 & 0.45 & 0.14 & 45.01 \\
\hline \multirow[t]{3}{*}{9} & Feb.20II & 8.3 & 12.4 & 7.04 & 33.77 & 36.7 & 3.42 & 0.62 & 139.13 \\
\hline & May.20II & 8.6 & 12.47 & 34.61 & 47.7 & 56.95 & 0.17 & 0.1 & 218.92 \\
\hline & Aug.20II & 8.34 & 4.91 & 36.79 & 81.1 & 45.65 & 0.76 & 0.59 & 159.59 \\
\hline \multirow[t]{3}{*}{10} & Feb.20II & 8 & 6.55 & 6.56 & 4.41 & 19.15 & 3.67 & 0.67 & 49.1 \\
\hline & May.20II & 8.8 & 10.53 & 13.55 & 64.91 & 77.65 & 0.34 & I.0I & 466.49 \\
\hline & Aug.2011 & 8.53 & 3.6 & 18.8 & 186.02 & 55.5 & 0.43 & 1.24 & 126.85 \\
\hline Station No. & $\begin{array}{l}\text { Sample } \\
\text { Date }\end{array}$ & $\begin{array}{l}\text { PH (6.5- } \\
8.5)\end{array}$ & $\begin{array}{l}\mathrm{DO}(>5) \\
\mathrm{mg} / \mathrm{l}\end{array}$ & $\begin{array}{l}\text { BOD (3-6) } \\
\mathrm{mg} / \mathrm{l}\end{array}$ & $\begin{array}{l}\text { CHL-A (5- } \\
\text { I40) ug/l }\end{array}$ & $\begin{array}{l}\text { TSM (25) } \\
\mathrm{mg} / \mathrm{l}\end{array}$ & $\begin{array}{l}\mathrm{NH} 4(0.4 \mathrm{I}) \\
\mathrm{mg} / \mathrm{l}\end{array}$ & $\begin{array}{l}\mathrm{NO3}(<0 . \mathrm{I}) \\
\mathrm{mg} / \mathrm{l}\end{array}$ & $\begin{array}{l}\text { TP(25- } \\
100) \text { ug/l } \\
\end{array}$ \\
\hline \multirow[t]{3}{*}{1} & Feb.2012 & 7.72 & 8.41 & 8.16 & 24.48 & 47.95 & 0.48 & 0.11 & 29.24 \\
\hline & May.20I2 & 8.18 & 7.34 & 21.05 & 8.83 & 70.2 & 0.06 & 1.97 & 97.46 \\
\hline & Aug.2012 & 8.7 & 6.52 & 59.22 & 6.1 & 38.55 & 0.42 & 0.02 & 77.16 \\
\hline \multirow[t]{3}{*}{2} & Feb.20I2 & 7.63 & 5.49 & 4.25 & 50.13 & 47.5 & 0.76 & 0.16 & 32.39 \\
\hline & May.20I2 & 8.16 & 7.9 & 21.77 & 19.21 & 62.3 & 0.09 & I.I4 & 96.45 \\
\hline & Aug. 2012 & 8.47 & 6.84 & 58.03 & 4.93 & 49.05 & 0.45 & 0.02 & 99.49 \\
\hline 3 & Feb.2012 & 7.71 & ND & 14.98 & 3.59 & 40.95 & 6.61 & 0.09 & 445.29 \\
\hline & May.20I2 & 7.65 & ND & 53.81 & 4.71 & 97.7 & 16.24 & 0.04 & 2431.52 \\
\hline & Aug.2012 & 8.61 & ND & 85.83 & 4.99 & 77.7 & 14.58 & 0.01 & 2557.41 \\
\hline 4 & Feb.20I2 & 7.15 & $4.4 I$ & 3.08 & 35.31 & 24.38 & $\mathrm{I} .7$ & 1.53 & 157.26 \\
\hline & May.20I2 & 7.84 & 4.47 & 8.61 & 27.64 & 132.6 & $\mathrm{I} .34$ & 1.49 & 632.5 \\
\hline & Aug.2012 & 8.75 & 5.96 & 54.45 & $|8.4|$ & 34.8 & 0.69 & 0.66 & 445.69 \\
\hline 5 & Feb.20I2 & 7.88 & 8.74 & 4.66 & $|88.2|$ & 37.85 & 2.5 & 0.84 & 243.76 \\
\hline & May.20I 2 & 8.58 & 7.34 & 10.53 & 253.11 & 112.8 & 0.06 & 0.28 & 834.54 \\
\hline & Aug.2012 & 8.45 & 5.09 & 52.06 & 53.86 & 24.65 & 0.23 & 0.33 & 299.5 \\
\hline 6 & Feb.2012 & 7.21 & 3.75 & 8.75 & 61.14 & 38.68 & 1.62 & 1.12 & 136.55 \\
\hline & May.20I 2 & 7.58 & ND & 41.98 & 92.25 & 46.85 & 3.34 & 1.09 & 824.38 \\
\hline & Aug.2012 & 8.55 & ND & 81.06 & I7.0| & 69.3 & 2.6 & 0.23 & 1609.17 \\
\hline 7 & Feb.2012 & 7.25 & 6.91 & 6.66 & 28.32 & 28.45 & 1.58 & $0.7 \mathrm{I}$ & 53.2 \\
\hline & May.20I 2 & 8.26 & 10.77 & 24.64 & 29.07 & 55.6 & 0.25 & 0.83 & 274.12 \\
\hline & Aug.2012 & 8.59 & 5.8 & 56.04 & 80.49 & 57.93 & 0.28 & 0.65 & 238.58 \\
\hline 8 & Feb.20I2 & 7.22 & 6.66 & 5.08 & 2.72 & 23.78 & 0.83 & 0.53 & 28.53 \\
\hline & May.20I 2 & 8.02 & 10.45 & 28.47 & 53.04 & 52.7 & 0.09 & 0.25 & 246.71 \\
\hline & Aug.2012 & 8.45 & 5.88 & 55.64 & 65.58 & 70.65 & 0.21 & 0.25 & 178.68 \\
\hline 9 & Feb.2012 & 7.21 & 5.08 & 4.33 & 27.86 & 20.3 & 1.2 & 1.01 & 92.9 \\
\hline & May.20I 2 & 8.11 & 11.56 & 31.34 & 33.91 & 72.55 & 0.15 & 0.56 & 188.84 \\
\hline & Aug.2012 & 8.63 & 6.04 & 58.03 & 33.56 & 44.33 & 0.52 & 0.08 & 137.06 \\
\hline 10 & Feb.2012 & 7.42 & 9.32 & 7.58 & 29.7 & 23.03 & 2.38 & 0.99 & 45.79 \\
\hline & May.20I 2 & 9.17 & 11.09 & 32.78 & 0.88 & 39.3 & 0.12 & 0.42 & 33.5 \\
\hline & Aug.2012 & 8.52 & 5.57 & 53.65 & 3.2 & 23.8 & 0.51 & 0.02 & 62.95 \\
\hline
\end{tabular}




\begin{tabular}{|c|c|c|c|c|c|c|c|c|c|}
\hline $\begin{array}{l}\text { Station } \\
\text { No. }\end{array}$ & $\begin{array}{l}\text { Sample } \\
\text { Date }\end{array}$ & $\begin{array}{l}\text { PH (6.5- } \\
8.5)\end{array}$ & $\begin{array}{l}\mathrm{DO}(>5) \\
\mathrm{mg} / \mathrm{l}\end{array}$ & $\begin{array}{l}\text { BOD (3- } \\
\text { 6) } \mathrm{mg} / \mathrm{l}\end{array}$ & $\begin{array}{l}\text { CHL-A (5- } \\
\text { I40) ug/I }\end{array}$ & $\begin{array}{l}\text { TSM (25) } \\
\text { mg/l }\end{array}$ & $\begin{array}{l}\mathrm{NH4}(0.4 \mathrm{I}) \\
\mathrm{mg} / \mathrm{l}\end{array}$ & $\begin{array}{l}\mathrm{NO3}(<0 . \mathrm{I}) \\
\mathrm{mg} / \mathrm{l}\end{array}$ & $\begin{array}{l}\text { TP(25-100) } \\
\text { ug/l }\end{array}$ \\
\hline \multirow[t]{3}{*}{ I } & Feb.20I3 & 8.6 & 14.22 & 8.41 & 42.97 & 49.1 & 0.07 & 0.25 & $|47.2|$ \\
\hline & May.2013 & 8.24 & 7.13 & 4.54 & 6.79 & 36.92 & 0.1 & 0.02 & $|00.5|$ \\
\hline & Aug.2013 & 8.34 & 11.77 & 5.97 & 10.58 & 54.95 & 0.13 & 0.06 & 67.01 \\
\hline \multirow[t]{3}{*}{2} & Feb.20I 3 & 8.64 & 12.28 & 8.1 & 47.79 & 49.75 & 0.05 & 0.93 & 116.75 \\
\hline & May.2013 & 8.12 & 6.16 & 3.4 & 3.13 & 37.03 & 0.11 & 0.02 & 79.19 \\
\hline & Aug.2013 & 8.32 & 9.06 & 5.64 & 9.84 & 45.05 & 0.13 & 0.11 & 89.34 \\
\hline \multirow[t]{3}{*}{3} & Feb.20। 3 & 7.68 & 0 & 38.72 & I.74 & 56.2 & 11.18 & 0.01 & 2572.64 \\
\hline & May.20I3 & 7.49 & 0 & 729 & 6.79 & 23.33 & 15.89 & 0.01 & 2234.57 \\
\hline & Aug. 2013 & 7.82 & ND & 63.6 & 4.59 & 21.8 & 8.71 & 0.04 & 1762.47 \\
\hline \multirow[t]{3}{*}{4} & Feb.20I3 & 7.9 & 7.28 & 9.26 & 2.72 & 23 & 0.08 & 0.9 & 546.2 \\
\hline & May.20I3 & 7.62 & 3.24 & 9.72 & 24.36 & 34.5 & 1.29 & 0.52 & 620.32 \\
\hline & Aug.2013 & 7.93 & 1.67 & 20.75 & 19.7 & 27.8 & 1.52 & 0.69 & 398.99 \\
\hline \multirow[t]{3}{*}{5} & Feb.2013 & 7.71 & 6.68 & 10.32 & 8.47 & 23.2 & 0.55 & 0.89 & 507.63 \\
\hline & May.20I3 & 7.67 & 4.21 & 7.78 & 27.5 & 22.43 & 0.2 & 0.21 & 453.82 \\
\hline & Aug. 2013 & 7.97 & 3.9 & 19.11 & 38.66 & 26.65 & 0.13 & 0.13 & 221.32 \\
\hline \multirow[t]{3}{*}{6} & Feb.2013 & 7.46 & 0 & 58 & 33.48 & 146.15 & 10.32 & 0.31 & 2253.86 \\
\hline & May.2013 & 7.6 & ND & 68.04 & 26.11 & 36.95 & 3.19 & 0.05 & 933.01 \\
\hline & Aug. 2013 & 7.8 & ND & 54.86 & 31.41 & 52.95 & 3.08 & 0.49 & 486.3 \\
\hline \multirow[t]{3}{*}{7} & Feb.20I3 & 8 & 9.01 & 2.94 & 25.01 & 31.95 & 0.14 & 0.42 & 93.4 \\
\hline & May.2013 & 8.27 & 8.59 & 9.72 & 32.62 & 38.5 & 1.96 & 0.02 & 278.18 \\
\hline & Aug.2013 & 8.4 & 12.72 & 12.24 & 6.07 & 44.75 & 0.15 & 0.4 & 141.12 \\
\hline \multirow[t]{3}{*}{8} & Feb.20I3 & 7.8 & 6.94 & 5.06 & 5.85 & 13.85 & 0.15 & 0.37 & 138.07 \\
\hline & May.2013 & 8.66 & 7.94 & 4.86 & 1.39 & 11.93 & 0.1 & 0.02 & 84.27 \\
\hline & Aug.2013 & 7.95 & 6.68 & 5.09 & 17.07 & 58 & 0.3 & 0.07 & 69.04 \\
\hline \multirow[t]{3}{*}{9} & Feb.20I3 & 8.34 & 10.75 & 4.07 & 58.43 & 39.3 & 0.25 & 0.42 & 184.78 \\
\hline & May.2013 & 8.56 & 8.42 & 20.41 & 67.87 & 47.2 & 0.11 & 0.02 & 240.61 \\
\hline & Aug.2013 & 7.96 & 6.68 & 1.27 & 6.1 & 22.55 & 0.16 & 0.07 & 40.61 \\
\hline \multirow[t]{3}{*}{10} & Feb.2013 & 8.05 & 13.75 & 3.87 & 13.4 & 34.05 & 0.37 & 0.66 & $\mid 40.1$ \\
\hline & May.2013 & 8.15 & 7.61 & 8.75 & 22.69 & 37.73 & 0.18 & 0.02 & $17 \mid .58$ \\
\hline & Aug.2013 & 7.99 & 5.72 & 5.08 & 41.18 & 18.85 & 0.11 & 0.04 & 127.92 \\
\hline $\begin{array}{l}\text { tation } \\
\text { Jo. }\end{array}$ & $\begin{array}{l}\text { Sample } \\
\text { Date }\end{array}$ & $\begin{array}{l}\text { PH (6.5- } \\
8.5)\end{array}$ & $\begin{array}{l}\mathrm{DO}(>5) \\
\mathrm{mg} / \mathrm{l}\end{array}$ & $\begin{array}{l}\text { BOD (3-6) } \\
\mathrm{mg} / \mathrm{l}\end{array}$ & $\begin{array}{l}\text { CHL-A (5- } \\
\text { I40) ug/I }\end{array}$ & $\begin{array}{l}\text { TSM (25) } \\
\mathrm{mg} / \mathrm{l}\end{array}$ & $\begin{array}{l}\mathrm{NH4(0.4I)} \\
\mathrm{mg} / \mathrm{l}\end{array}$ & $\begin{array}{l}\mathrm{NO3}(<0.1) \\
\mathrm{mg} / \mathrm{l}\end{array}$ & $\begin{array}{l}\text { TP(25-100) } \\
\text { ug/l }\end{array}$ \\
\hline \multirow[t]{3}{*}{ I } & Feb.20I4 & 8.03 & 6.83 & 31.68 & 8.94 & 48 & 0.05 & 0.08 & 183.23 \\
\hline & May.20I4 & 8.2 & 4.63 & 22.76 & 43.94 & 87.8 & 0.15 & 0.06 & 135.03 \\
\hline & Aug.2014 & 9.04 & 5.93 & 30.3 & 12.14 & 73.2 & 0.49 & 0.63 & 123.88 \\
\hline \multirow[t]{3}{*}{2} & Feb.20I4 & 8.23 & 7.07 & 23.97 & 0.86 & 32.9 & 0.08 & 0.02 & 252.05 \\
\hline & May.20I4 & 8.15 & 4.96 & 19.84 & 30.29 & 68.4 & 0.16 & 0.07 & $|20.8|$ \\
\hline & Aug.2014 & 9 & 5.93 & 30.3 & 11.62 & 59.45 & 0.51 & 0.24 & 125.6 \\
\hline \multirow[t]{3}{*}{3} & Feb.20I4 & 7.56 & 0 & 120.48 & 7.08 & 33.55 & 20.34 & 0.01 & | 373.82 \\
\hline & May.20I4 & 7.51 & 0 & 620.48 & 6.74 & 30.35 & 7.15 & 0.07 & 2385.84 \\
\hline & Aug.2014 & 8.5 & 0 & 107.23 & 2.22 & 35.4 & 9.04 & 0.01 & 1933.84 \\
\hline & Feb.2014 & 7.7 & 2.28 & 61.71 & 4.47 & 16.85 & 2.65 & 0.42 & 499.81 \\
\hline & May.20I4 & 7.7 & 0 & 222.5 & 66.58 & 27.4 & 0.53 & 0.83 & 309.65 \\
\hline & Aug.2014 & 8.75 & 2.03 & 24.87 & 19.69 & 37.5 & 1.4 & 1.37 & 450.77 \\
\hline & Feb.20I4 & 7.99 & 4.23 & 13.49 & 133.29 & 35.4 & 0.28 & 0.22 & 480.02 \\
\hline & May.20I4 & 8.08 & 7.8 & 17.59 & 225.09 & 26.65 & 0.14 & 0.3 & 514.73 \\
\hline & Aug.2014 & 9.05 & 13 & 39.98 & 216.1 & 47.7 & 0.58 & 0.16 & 294.21 \\
\hline & Feb.2014 & 7.7 & 0 & 136.72 & 82.44 & 62.54 & 4.78 & 0.43 & 54I.I \\
\hline & May.20I4 & 7.67 & 0 & 593.01 & 80.8 & 27.85 & 1.15 & 0.46 & 924.89 \\
\hline & Aug.2014 & 8.58 & 0 & 94.73 & 21.96 & 46.75 & 3.8 & 0.55 & 629.7 \\
\hline & Feb.20I4 & 8.28 & 9.75 & 17.11 & 20.72 & 37.75 & 0.21 & 0.05 & 184.95 \\
\hline & May.20I4 & 8.1 & 7.15 & 17.15 & 23.27 & 37.7 & 0.13 & 0.05 & 131.98 \\
\hline & Aug.2014 & 8.88 & 7.72 & 31.85 & 4.02 & 35.05 & 0.2 & 0.07 & 81.72 \\
\hline & Feb.20I4 & 8.2 & 9.59 & 18.22 & 102.77 & 41.35 & 0.09 & 0.26 & 471.42 \\
\hline & May.20I4 & 8.37 & 9.83 & 28.3 & 41.05 & 17.45 & 0.11 & 0.02 & 216.25 \\
\hline & Aug.2014 & 8.85 & 8.05 & 33.29 & 33.67 & 56.3 & 0.24 & 0.02 & 215.92 \\
\hline & Feb.20l4 & 8.26 & 11.95 & 22.49 & 78.58 & 43.25 & 0.75 & 0.45 & 443.03 \\
\hline & May.20I4 & 8.38 & 8.53 & 21.78 & 139.92 & 39.1 & 0.27 & 0.19 & 322.85 \\
\hline & Aug.2014 & 9.05 & 12.43 & 38.1 & 138.34 & 38.15 & 0.35 & 0.33 & 196.14 \\
\hline 0 & Feb.2014 & 8.13 & 9.51 & 8.92 & $4.4 I$ & 23.85 & 0.06 & 0.1 & 109.25 \\
\hline & May.20I4 & 8.26 & 3.66 & 19.89 & 3.54 & 19.35 & 0.05 & 0.04 & 38.58 \\
\hline & Aug.20I4 & 8.71 & 2.68 & 24.75 & 3.55 & 13.55 & 0.32 & 0.09 & 55.92 \\
\hline
\end{tabular}

Note: Bold value is out of EEAA standard. 
Table 4 Summary of CWQI Factors for the Ten Stations in Mariout Lake (a) 2010 , (b) 20II, (c) 20I2, (d) 20I3, (e) 20I4

\begin{tabular}{|c|c|c|c|c|c|}
\hline \multicolumn{6}{|l|}{ CWQI-20I0 } \\
\hline Station No & FI & $\mathbf{F 2}$ & NSE & F3 & CWQI \\
\hline I & 62.5 & 58.33 & 1.12 & 52.85 & 41.97 \\
\hline 2 & 50 & 41.67 & 0.85 & 45.91 & 54.01 \\
\hline 3 & 87.5 & 79.17 & 14.06 & 93.36 & 13.13 \\
\hline 4 & 100 & 79.17 & 3.84 & 79.34 & 13.27 \\
\hline 5 & 87.5 & 58.33 & 2.5 & 71.39 & 26.61 \\
\hline 6 & 87.5 & 70.83 & 2.53 & 71.64 & 22.96 \\
\hline 7 & 87.5 & 79.17 & $1.6 \mathrm{I}$ & 61.74 & 23.11 \\
\hline 8 & 100 & 66.67 & 2.03 & 67 & 20.56 \\
\hline 9 & 87.5 & 54.17 & 4.29 & 81.09 & 24.35 \\
\hline 10 & 75 & 58.33 & 1.85 & 64.94 & 33.55 \\
\hline \multicolumn{6}{|l|}{ CWQI-20I I } \\
\hline Station No. & F I & $\mathbf{F 2}$ & NSE & F3 & CWQI \\
\hline 1 & 75 & 45.83 & 0.88 & 46.7 & 42.53 \\
\hline 2 & 75 & 45.83 & 0.53 & 34.55 & 45.47 \\
\hline 3 & 87.5 & 70.83 & 5.45 & 84.49 & 18.73 \\
\hline 4 & 87.5 & 75 & 2.74 & 73.3 & 21.14 \\
\hline 5 & 87.5 & 75 & 2.7 & 72.95 & 21.25 \\
\hline 6 & 75 & 75 & 4.17 & 80.64 & 23.07 \\
\hline 7 & 87.5 & 70.83 & 1.87 & 65.13 & 24.91 \\
\hline 8 & 75 & 54.17 & 2.11 & 67.89 & 33.75 \\
\hline 9 & 87.5 & 62.5 & 1.38 & 58 & 29.46 \\
\hline 10 & 100 & 66.67 & 1.91 & 65.63 & 20.94 \\
\hline
\end{tabular}

\begin{tabular}{llllll}
\hline CWQI-20I2 & & & & & \\
\hline Station No. & FI & F2 & NSE & F3 & CWQI \\
\hline I & 62.5 & 45.83 & I.42 & 58.64 & 43.89 \\
2 & 75 & 41.67 & I.II & 52.59 & 41.9 \\
3 & 85.71 & 61.9 & 6.57 & 86.79 & 21.02 \\
4 & 87.5 & 66.67 & $2.6 I$ & 72.33 & 23.99 \\
5 & 87.5 & 58.33 & 1.76 & 63.79 & 28.99 \\
6 & 87.5 & 54.17 & 3.42 & 77.36 & 19.15 \\
7 & 75 & 54.17 & 1.7 & 63 & 35.38 \\
8 & 75 & 45.83 & 1.1 & 52.37 & 40.93 \\
9 & 75 & 45.83 & 1.36 & 57.7 & 39.29 \\
10 & 75 & 45.83 & 1.29 & 56.29 & 39.74 \\
\hline
\end{tabular}

\begin{tabular}{llllll}
\multicolumn{6}{l}{ CWQI-20 I 3 } \\
Station & F I & F2 & NSE & F3 & CWQI \\
\hline I & 62.5 & 33.33 & 0.21 & 17.33 & 57.9 \\
2 & 75 & 37.5 & 0.49 & 32.94 & 47.99 \\
3 & 75 & 60.87 & 11.9 & 92.25 & 22.88 \\
4 & 87.5 & 66.67 & 1.8 & 64.22 & 26.46 \\
5 & 75 & 54.17 & 0.92 & 48.01 & 39.82 \\
6 & 75 & 68.18 & 4.62 & 82.19 & 24.65 \\
7 & 62.5 & 37.5 & 0.63 & 38.48 & 52.41 \\
8 & 62.5 & 20.83 & 0.29 & 22.62 & 59.78 \\
\hline
\end{tabular}

\begin{tabular}{llllll}
\hline \multicolumn{6}{l}{ CWQI-20 I 4 } \\
Station & FI & F2 & NSE & F3 & CWQI \\
\hline I & 87.5 & 54.17 & 0.98 & 49.52 & 34.06 \\
2 & 100 & 58.33 & 0.89 & 47.01 & 27.86 \\
3 & 75 & 66.67 & 11.69 & 92.12 & 21.35 \\
4 & 100 & 79.17 & 3.87 & 79.47 & 13.24 \\
5 & 100 & 79.17 & 1.07 & 51.74 & 20.53 \\
6 & 87.5 & 79.17 & 7.79 & 88.63 & 14.8 \\
7 & 62.5 & 41.67 & 0.45 & 31.22 & 53.03 \\
8 & 62.5 & 41.67 & 0.83 & 45.31 & 49.35 \\
\hline
\end{tabular}

Table 5 Showing CWQI Scores and Rankings for Ten Stations in Mariout Lake during 20I0 -20I4

\begin{tabular}{|c|c|c|c|c|c|c|c|c|c|c|}
\hline $\begin{array}{l}\text { Station } \\
\text { No. }\end{array}$ & $\begin{array}{l}\text { CWQI } \\
2010\end{array}$ & $\begin{array}{l}\text { Rating } \\
2010\end{array}$ & $\begin{array}{l}\text { CWQI } \\
201 \text { I }\end{array}$ & $\begin{array}{l}\text { Rating } \\
20 \text { II }\end{array}$ & $\begin{array}{l}\text { CWQI } \\
2012\end{array}$ & $\begin{array}{l}\text { Rating } \\
2012\end{array}$ & $\begin{array}{l}\text { CWQI } \\
2013\end{array}$ & $\begin{array}{l}\text { Rating } \\
2013\end{array}$ & $\begin{array}{l}\text { CWQI } \\
2014\end{array}$ & $\begin{array}{l}\text { Rating } \\
2014\end{array}$ \\
\hline I & 41.97 & Poor & 42.53 & Poor & 43.89 & Poor & 57.9 & Marginal & 34.06 & Poor \\
\hline 2 & 54.01 & Marginal & 45.47 & Marginal & 41.9 & Poor & 47.99 & Marginal & 27.86 & Poor \\
\hline 3 & 13.13 & Poor & 18.73 & Poor & 21.02 & Poor & 22.88 & Poor & 21.35 & Poor \\
\hline 4 & 13.27 & Poor & 21.14 & Poor & 23.99 & Poor & 26.46 & Poor & 13.24 & Poor \\
\hline 5 & 26.61 & Poor & 21.25 & Poor & 28.99 & Poor & 39.82 & Poor & 20.53 & Poor \\
\hline 6 & 22.96 & Poor & 23.07 & Poor & 19.15 & Poor & 24.65 & Poor & 14.8 & Poor \\
\hline 7 & 23.11 & Poor & 24.91 & Poor & 35.38 & Poor & 52.41 & Marginal & 53.03 & Marginal \\
\hline 8 & 20.56 & Poor & 33.75 & Poor & 40.93 & Poor & 59.78 & Marginal & 49.35 & Marginal \\
\hline 9 & 24.35 & Poor & 29.46 & Poor & 39.29 & Poor & 55.4 & Marginal & 37.39 & Poor \\
\hline 10 & 33.55 & Poor & 20.94 & Poor & 39.74 & Poor & 63.42 & Marginal & 54.19 & Marginal \\
\hline
\end{tabular}

Table 6 Water and aquatic vegetation area of each date in Mariout Lake

\begin{tabular}{lll}
\hline Image date & Water $\left(\mathbf{k m}^{2}\right)$ & Vegetation $\left.\mathbf{( k m}^{2}\right)$ \\
\hline $19-09-1972$ & 66.1 & 7.26 \\
$11 / 9 / 1984$ & 34.32 & 36.9 \\
$18-06-1997$ & 11.39 & 57.73 \\
$23-12-200 \mid$ & 27.51 & 38.76 \\
$19-03-2006$ & 26.83 & 37.03 \\
$4 / 5 / 2009$ & 20.58 & 42.01 \\
$28-01-2014$ & 18.34 & 44.17 \\
$12 / 2 / 2016$ & 26.94 & 35.13
\end{tabular}

Nitrate concentration on ten stations ranging $0.01-3.66 \mathrm{mg} / \mathrm{l}$. This highly related to the huge number of human activities nearby. Major source of river water pollution comes from domestic sewage, animal waste, agricultural waste, soil erosion and runoff from the settlement. ${ }^{17}$ EEAA quality standards, nitrate content has to be $\leq 0.1$ $\mathrm{mg} / \mathrm{l}$, Station 3 recorded low Nitrate $(0.01)$, and station 4 recorded high Nitrate (3.66). Therefore nitrate at most stations was out of the quality standard.

Phosphate concentration gives an indication of the nutrient levels and eutrophication of the lake system. ${ }^{18}$ Total phosphate concentration in Mariout Lake ranged $24.55-3470.02 \mathrm{mg} / 1$. Total phosphate in all stations was high. This denoted the relatively worst condition of the 
nearby lake. Human activities affected significantly on phosphate concentration. The source of phosphate in water is derived from the weathering of rock minerals, decomposition of organic matter, detergents, fertilizers, industrial waste, household waste, and phosphate minerals. EEAA quality standards, total phosphate content must be $\leq 100 \mathrm{mg} / \mathrm{l}$. Therefore, total phosphate on ten stations was out of the quality standard.

\section{Surface water detection}

\section{Calculation of NDWI}

The Normalized Difference Water Index (NDWI) was first proposed by McFeeters in 1996 to detect surface waters in wetland environments and to allow for the measurement of surface water extent, ${ }^{19}$ and it produces a single grayscale image, where water is bright. Although the index was created for use with different images data, it has been successfully used with other sensor systems in applications where the measurement of the extent of open water is needed. ${ }^{20,21}$ The NDWI is calculated using following Equation 9:

$$
N D W I=\frac{(\text { Band } 2-\text { Band } 4)}{(\text { Band } 2+\text { Band } 4)} 9
$$

Where, Band 2 is the TOA green light reflectance and Band 4 is the TOA near-infrared (NIR) reflectance. McFeeters ${ }^{19}$ asserted that values of NDWI greater than zero are assumed to represent water surfaces, while values less than, or equal, to zero are assumed to be nonwater surfaces. Values of NDWI were calculated from the Satellite images using Equation (9) in the Raster Calculator tool in the Spatial Analyst ${ }^{\circledR}$ extension in ArcGIS 10.3. The result was a single band gray-scale floating point grid file that was color-coded to facilitate analysis as shown in Figure 4.

\section{Conclusion}

Based on the CCME-WQI during the period from 2010 to 2014, the water quality can be categorized as "Poor". However, all parameters reached out of the EEAA quality standards.

NDWI indicated that change in Aquatic Vegetation for all basins increased by $27.68 \mathrm{~km}^{2}$, and the changes in water for all basins decreased by $38.67 \mathrm{~km}^{2}$ during the study period. NDWI indicated that change in water for each basin that main basin decreased by $10.82 \mathrm{~km}^{2}$, fisheries basin decreased by $1.38 \mathrm{~km}^{2}$, northwest decreased by $2.5 \mathrm{~km}^{2}$, and southwest decreased by $23.96 \mathrm{~km}^{2}$. NDWI indicated that change in aquatic vegetation for each basin that main basin increased by $4.81 \mathrm{~km}^{2}$, fisheries basin increased by $0.286 \mathrm{~km}^{2}$, northwest increased by $1.84 \mathrm{~km}^{2}$, and southwest increased by $20.75 \mathrm{~km}^{2}$.

CWQI conditions in Mariout Lake have been classified as poor. However, there are all parameters reaching out of the EEAA quality standards. This condition showed that the activities of the people and micro-hydro had negative effect on the water quality of basins in mariout Lake. Samples at 10 stations in the year 2010 - 2014 from Mariout Lake have been investigated. Datasets obtained have been converted into simple maps using coastal water quality index (CWQI) and Geographical Information System (GIS) based overlay mapping technique to demarcate healthy and polluted areas. Analysis of multiple parameters revealed poor water quality in Main Basin and Northwest Basin. The anthropogenic activities may be the likely cause for poor water quality. Whereas, good water quality was witnessed at Fish basin and Southwest Basin. This study is an attempt to integrate CWQI and GIS-based mapping technique to derive a reliable, simple and useful output for water quality monitoring in coastal environment lakes.

\section{Suggestions}

Based on the results of this study to control and reduce water quality following is recommended:

1. Activation of municipal and industrial wastewater treatment plant standard discharge to surface water.

2. Entering the aquacultures waste water effluent monitoring with EEAA standards for surface water discharge.

\section{Acknowledgments}

None.

\section{Conflicts of interest}

The authors declare there is no conflict of interests.

\section{Funding}

None.

\section{References}

1. Lumb A, Halliwell D, Sharma T. Application of CCME water quality index to monitor water quality: A case study of the Mackenzie River basin, Canada. Environmental Monitoring and Assessment. 2006;113(13):411-429.

2. Gyamfi C, Boakye R, Awuah E, et al. Application of the Ccme-Wqi model in assessing the water quality of the Aboabo River, KumasiGhana. Journal of Sustainable Development. 2013;6(10):1.

3. El-Sadek A, Feyen J, Berlamont J. Comparison of models for computing drainage discharge. Journal of irrigation and drainage engineering. 2001;127(6):363-369.

4. Radwan M. River water quality modelling as water resources management tool at catchment scale (Doctoral dissertation, $\mathrm{PhD}$ Dissertation, Faculty of Engineering, Katholieke Universiteit Leuven, Belgium); 2002

5. Saksena DN, Garg RK, Rao RJ. Water quality and pollution status of Chambal river in National Chambal sanctuary, Madhya Pradesh. Journal of Environmental Biology. 2008;29(5):701-710.

6. Effendi H. Water quality analysis for aquatic resources management. Yogyakarta: Kanisius; 2003.

7. Said A, Stevens DK, Sehlke G. An innovative index for evaluating water quality in streams. Environmental management. 2004;34(3):406-414.

8. Ott WR. Environmental indices:theory and practice. 1978.

9. Buchari AI, Putra KGD, Dewi I. Environmental chemistry. 2001.

10. APHA-AWWA-WPCF. Standard methods for the examination of water and wastewater. APHA American Public Health Association; 1981.

11. Fatoki OS, Muyima NYO, Lujiza N. Situation analysis of water quality in the Umtata River catchment. Water. SA 2001;27(4):467-474.

12. Dihkan M, Karsli F, Guneroglu A. Mapping total suspended matter concentrations in the Black Sea using Landsat TM multispectral satellite imagery. Fresenius Environmental Bulletin. 2011;20(1 A):262-269.

13. Grove MK, Bilotta GS, Woockman RR, et al. Suspended sediment regimes in contrasting reference-condition freshwater ecosystems:Implications for water quality guidelines and management. Science of the Total Environment. 2015;502:481-492.

14. Cerco CF, Kim SC, Noel MR. Management modeling of suspended solids in the Chesapeake Bay, USA. Estuarine, Coastal and Shelf Science. 2013;116:87-98. 
15. Skarbøvik E, Stålnacke P, Bogen J, et al. Impact of sampling frequency on mean concentrations and estimated loads of suspended sediment in a Norwegian river:implications for water management. Science of the Total Environment. 2012;433:462-471.

16. Senila M, Levei E, Miclean M, et al. Study regarding the water quality in Aries catchment. Romania: Babes-Bolyai University; 2007.

17. Christensen VG, Lee KE, McLees JM, et al. Relations between retired agricultural land, water quality, and aquatic-community health, Minnesota River Basin. Journal of environmental quality. 2012;41(5):1459-1472.

18. Heath R, Brown C. Orange river integrated water resources management plan. Environmental Considerations Pertaining to the Orange River
Unpubl Report, WRP Consulting Engineers, Jeffares and Green, Sechaba Consulting, WCE Pty Ltd, Water Surveys Botswana (Pty) Ltd; 2007. 190 p.

19. McFeeters SK. The use of the Normalized Difference Water Index (NDWI) in the delineation of open water features. International Journal of remote sensing. 1996;17(7):1425-1432.

20. Chowdary VM, Chandran RV, Neeti N, et al. Assessment of surface and sub-surface waterlogged areas in irrigation command areas of Bihar state using remote sensing and GIS. Agricultural water management. 2008;95(7):754-766.

21. US Geological Survey (USGS) and US Department of the Interior. Normalized Difference Water Index (NDWI) 